\title{
EFFECTS OF FOAM ROLLING ON BLOOD LACTATE CONCENTRATION IN ELITE FUTSAL PLAYERS
}

original paper

( ) University School of Physical Education in Wroclaw

DOI: https://doi.org/10.5114/hm.2021.98467

\section{RAMDAN PELANA ${ }^{1}$, TOMMY APRIANTONO ${ }^{2}$, BAGUS WINATA BAGUS ${ }^{2}$, AGUNG DWI JUNIARSYAH ${ }^{2}$, SRI INDAH IHSANI ${ }^{2}$}

\author{
${ }^{1}$ State University of Jakarta, Jakarta, Indonesia \\ ${ }^{2}$ Bandung Institute of Technology, Bandung, Indonesia
}

\section{ABSTRACT}

Purpose. Foam rolling (FR) is a known recovery method based on self-myofascial release. Detailed data on FR to reduce muscle fatigue and enhance performance in futsal athletes are still limited. The study aimed to assess whether FR was a suitable recovery method for futsal athletes to enhance performance and reduce their lactic acid concentration.

Methods. A total of 30 male futsal athletes participated in this study. They were randomly divided into 2 groups: 15 subjects assigned to the experimental (FR) group practised foam roller exercises, while 15 individuals assigned to the control group were given slow jogging recovery. Both groups completed pre- and post-recovery tests for anaerobic capacity and agility. Blood lactate was measured before the experiment, immediately after the tests, and immediately after recovery. A $2 \times 2$ repeated measures analysis of variance (ANOVA) was used to calculate each parameter results.

Results. In the post-recovery lactate concentration, the independent t-test revealed a significant decrease between pre- and post-test values in the FR group $(p=0.033)$. Conversely, an increase in lactate between pre- and post-test measurements occurred in the control group $(p=0.058)$. Furthermore, ANOVA revealed significant time $\times$ group interactions $(p=0.002)$ and a significant main effect for group $(p=0.001)$.

Conclusions. FR after exercise-induced muscle fatigue had an effect on the recovery of elite futsal players by increasing the rate of blood lactate clearance. There was no increase in performance in 20-m sprint or agility ( $t$-test).

Key words: lactate, muscle function, muscle soreness, pain, performance, recovery

\section{Introduction}

Futsal is five-a-side indoor football and has become very popular in recent years, with 12 million players in over 100 countries [1]. Match analysis and physiological monitoring show that futsal is characterized by intermittent high-intensity movements [2]. Additionally, previous studies revealed that in $46 \%$ of the total distance and play time, professional futsal players perform movement on the field with an average intensity of more than $80 \%$ of maximal oxygen uptake (high intensity) [3]. Thus, coaches and futsal athletes are continuously looking for ways to influence the body physiological systems with designing a heavy training system, so that athletes could better cope with acute fatigue [4].
Some studies have revealed that fatigue can be a problem for athletes because, in addition to reducing performance, it is also known to increase the risk of injury [5, 6]. Furthermore, previous literature implies that fatigue is a phenomenon of accumulation of lactic acid in muscle fibres, caused by high-intensity training [7, 8]. Because of this phenomenon, technical and medical staff must try to find the right and effective recovery method to reduce lactic acid concentration quickly, so that all athletes can reinvent or improve their performance [9]. Also, it is common for the technical and medical staff to use a variety of different post-exercise strategies in order to accelerate the recovery process and reduce the incidence of fatigue [10].

Experts now divide recovery into the categories of active and passive recovery [11]. Several recovery

Correspondence address: Ramdan Pelana, Universitas Negeri Jakarta, Faculty of Sports Sciences, Rawamangun Muka Street, RT.11/RW.14, Rawamangun, Kec. Pulo Gadung, East Jakarta City, Jakarta Capital City, Indonesia, 13220, e-mail: ramdanpelana@unj.ac.id

Received: September 2, 2019

Accepted for publication: February 24, 2020

Citation: Pelana R, Apriantono T, Bagus BW, Juniarsyah AD, Ihsani SI. Effects of foam rolling on blood lactate concentration in elite futsal players. Hum Mov. 2021;22(1):72-79; doi: https://doi.org/10.5114/hm.2021.98467. 
methods have been observed to be beneficial and efficient in futsal; these include light-emitting diode therapy, water immersion (cold, hot, thermoneutral, or contrast water), and low-intensity aerobic activity [12-14]. While a few recovery methods in futsal athletes are relatively well documented in many studies, detailed data on foam rolling (FR) to reduce muscle fatigue and enhance performance in this group are still limited. FR is a known recovery method based on self-myofascial release [15]. The idea is that the mechanical technique that mimics therapeutic massage in FR may have recovery benefits to reduce thickening, adhesion, tension of fascial tissue and muscle, as well as to enhance sports performance [16].

Some research has suggested that FR has no benefits for recovery. Specifically, Healey et al. [17] imply that FR has no effect on athletic performance despite reducing post-exercise fatigue. However, many previous studies also revealed FR efficacy for athletes' recovery [18-20]. For example, Macdonald et al. [21] explained that FR was better at reducing muscle soreness than passive recovery and it could improve vertical jump height, muscle activation, and passive and dynamic range of motion. Recently, Rey et al. [18] established that FR was advantageous for soccer athletes who did 5 FR exercises on the body parts often engaged in playing football (quadriceps, hamstrings, adductors, gluteal, and gastrocnemius). While these studies present equivocal findings concerning performance improvement after FR application, physiological measurements of blood lactate are lacking.

In the context of previous problems related to the advantages and disadvantages of using FR, this study aimed to assess whether FR was a suitable recovery method for futsal athletes to enhance performance and reduce lactic acid concentration. To the authors' best knowledge, no available studies have reported the effect of FR to reduce blood lactate and enhance performance in futsal athletes. We hypothesized that recovery involving FR might benefit to reduce lactate concentration and enhance performance in futsal athletes.

\section{Material and methods}

\section{Subjects}

The study involved 30 Indonesian futsal athletes from the ITB Futsal Club. Before their inclusion, the authors had to ensure that the athletes had no history of smoking. Other inclusion criteria were the following: a minimum of 5 years of futsal training experience and participation in systemized training with a minimum duration of 3 training sessions per week, with each training session of at least 3 hours. Those who had a cardiovascular disease or a history of a musculoskeletal injury 2 months prior to the study commencement were excluded. The study also established rules for all participants not to consume alcohol and caffeine for 24 hours prior to the experiment. We also ensured that all participants had no experience of FR exercises. Table 1 provides the anthropometric characteristics of the subjects. All participants received an explanation of the procedures and risks of the study.

Table 1. Anthropometric characteristics of participants

\begin{tabular}{lrr}
\hline Variables & $\begin{array}{c}\text { FR group } \\
(\bar{x} \pm S D)\end{array}$ & \multicolumn{1}{c}{$\begin{array}{c}\text { SJR group } \\
(\bar{x} \pm S D)\end{array}$} \\
\hline Age (years) & $21.67 \pm 0.72$ & $21.80 \pm 1.01$ \\
Weight $(\mathrm{kg})$ & $69.66 \pm 6.21$ & $68.50 \pm 4.22$ \\
Height $(\mathrm{cm})$ & $176.47 \pm 6.03$ & $175.93 \pm 4.77$ \\
BMI $\left(\mathrm{kg} \cdot \mathrm{m}^{-2}\right)$ & $22.37 \pm 1.62$ & $22.14 \pm 1.19$ \\
\hline
\end{tabular}

FR - foam rolling, SJR - slow jogging recovery, BMI - body mass index

\section{Study design}

To determine the effect of the independent variable on the dependent variables, the study used a parallel, 2-group, purposive sampling, pretest-posttest design. The independent variable was FR, while the dependent variables included blood lactate concentration, agility, and anaerobic performance of the athletes. All subjects were randomly divided into 2 groups: 15 individuals assigned to the experimental (FR) group practised FR exercises, while 15 subjects assigned to the control group received slow jogging recovery (SJR). Both groups were required to complete pre- and post-recovery tests for anaerobic capacity and agility. Additionally, blood lactate was measured before and immediately after the test and immediately after recovery. The research design is illustrated in Figure 1.

\section{Protocol procedures}

In the study, 24 hours prior to the experiment commencement, all participants were prohibited from engaging in strenuous physical activity; also, they did not consume any food for 3 hours before the start of the test. The testing began with anthropometric measurements, performed in a laboratory. We used Omron HN 289 digital weight scales to measure body weight, with the participants wearing only thin pants and barefoot. A Seca 214 portable stadiometer (Cardinal Health, 


\section{HUMAN MOVEMENT}

R. Pelana et al., Effects of foam rolling in futsal players

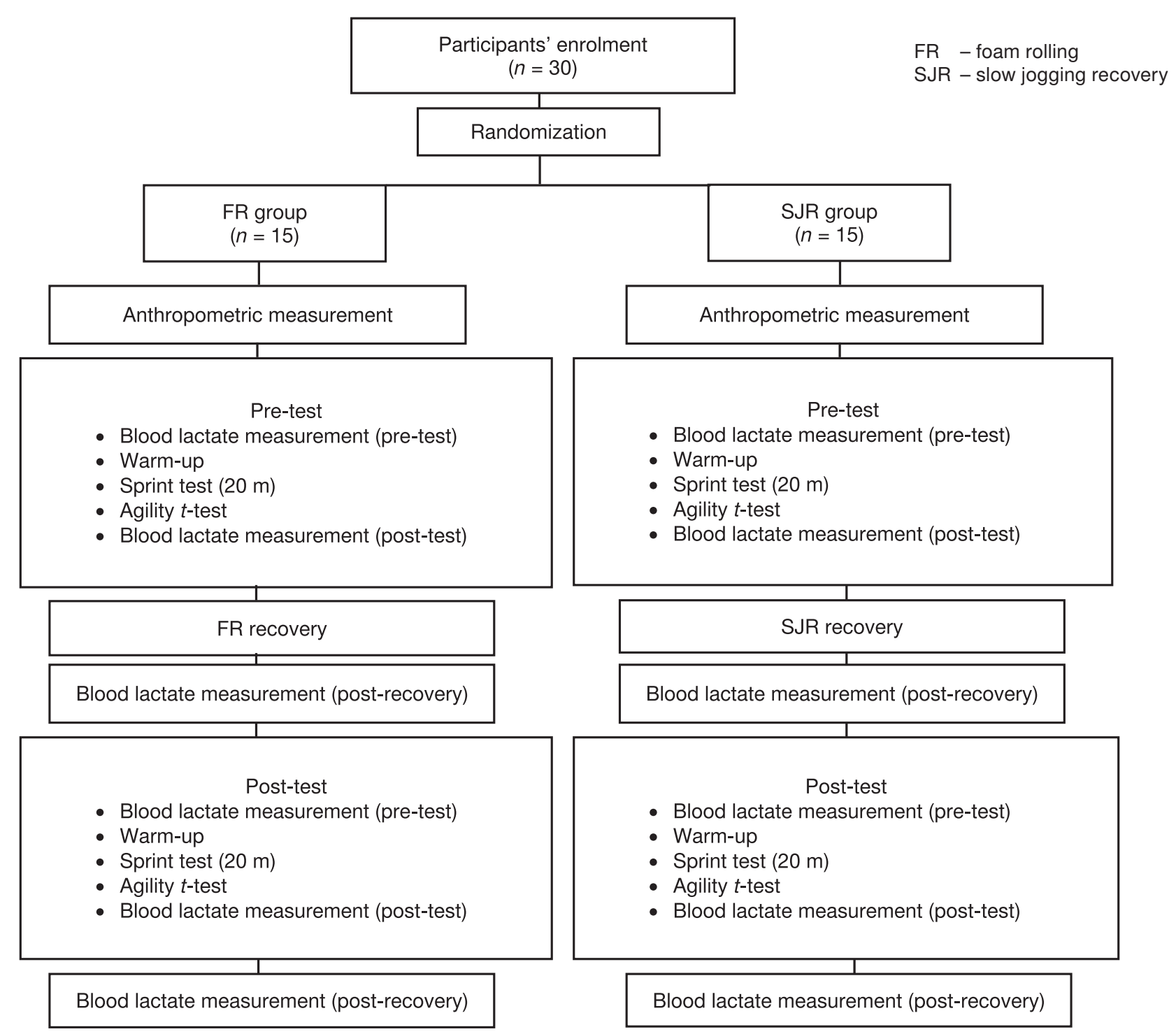

Figure 1. The research design

Dublin, USA) served to determine the subjects' body height in accordance with established procedures. Body mass index was calculated as body mass $(\mathrm{kg})$ divided by body height (m) squared.

After all participants completed the anthropometric measurement session, they prepared to the pre-test lactate assessment. In this evaluation, we used a Lactate Pro analyser (Arkray, Kōka, Japan); a 100- $\mathrm{l}$ sample of fingertip capillary blood was collected from each participant for the analysis. Before starting the pre-test session, all subjects were required to do jogging at $6.8 \mathrm{~km} \cdot \mathrm{h}^{-1}$ as a form of warm-up. Pre-test measurements included an anaerobic capacity test (20-m sprint test) and an agility $t$-test, which started at 09:30 a.m., with a 10-s break between the tests. Blood lactate was determined immediately, after each participant completed their assignment. After the blood lactate measurements, the athletes were assigned to the FR or SJR group to get respective recovery.

A high-density foam roller was used in the FR group. The intervention consisted of 5 different exercises, targeting muscle groups most used in futsal, applied for $1 \mathrm{~min}$ on each area (quadriceps, hamstrings, adductors, gluteal, and gastrocnemius). The SJR group performed active recovery, namely jogging at a speed of $6.8 \mathrm{~km} \cdot \mathrm{h}^{-1}$ for $8 \mathrm{~min}$. Verbal feedback from lap times was used to control the jogging speed of each participant. After the recovery session, blood lactate was determined immediately. During the recovery period, all subjects were allowed to drink mineral water ad libitum. They were also required to sleep from 09:00 p.m. to 6:00 a.m. The post-testing measurements were identical to baseline testing and initiated 24 hours after the end of pre-testing. 


\section{Sprint test}

A 20-m 'maximal' running sprint was performed in the Saraga athletic stadium. Before the sprint, the participants were instructed to run as fast as possible. Two cones were installed at the beginning (cone A) and at the end of the track (cone B). The first administrator was in charge of giving the signal ' $3-2-1-G o$ '. On the 'Go' signal, the participants ran as fast as possible towards the finish line. When a subject crossed the finish line, the second administrator, near cone $\mathrm{B}$, shouted 'Clear' as a sign that the test had finished, in accordance with the procedure. A beam photocell system (Microgate, Bolzano, Italy) was used in the study to record the running times.

\section{Agility t-test}

The test involved 4 cones ( $5 \mathrm{~m}$ apart). Each participant was to run to the cone in question, then touch the cone, and go directly to another cone. Administrators counted down to the start of the test ('3-2-1-Go'). On the 'Go' signal, the test administrator pressed the start button on the stopwatch and the participant went straight to cone B. After reaching cone B, they immediately turned left and moved sideways towards cone $\mathrm{C}$. After touching cone $\mathrm{C}$, they moved to the right side and headed to cone $\mathrm{D}$. They then retreated to cone $\mathrm{B}$, touched it with their left hand, and then ran backwards to cone A. The stopwatch was stopped as the subject passed cone A. The test was considered a failure if the participant crossed one foot in front of the other while shuffling, did not touch the cone properly, or failed to face forward throughout the test. The better time of 2 successful trials was recorded to the nearest $0.1 \mathrm{~s}$. All times were recorded with a stopwatch (Seiko stopwatch S23601P).

\section{Foam rolling recovery}

After the final assignment in pre- and post-testing, recovery protocols were implemented immediately. FR was performed with the Grid Foam Roller (Kettler) $(14 \times 33 \mathrm{~cm})$. The FR protocol was based on previous research [18]. FR consisted of 5 exercises, for quadriceps, hamstrings, adductors, gluteal, and gastrocnemius, with $1 \mathrm{~min}$ for each exercise and $15 \mathrm{~s}$ for rest. All participants started with placing a foam roller at the most distal portion of the muscle; they put as much of their body mass as tolerable on the foam roller at all times. With a rhythm of 50 beats per minute, the athletes were instructed to roll their body mass across the foam roller [20]. An investigator was responsible for supervising the FR in the subjects in accordance with their assignment.

\section{Statistical analysis}

The values are presented as mean $\pm S D$. The Shapiro-Wilk test was used to verify the normality of all sample data distribution. A 2 (group: FR and SJR groups) $\times 2$ (time: pre- and post-test) repeated measures analysis of variance (ANOVA) was applied for each parameter. An independent $t$-test served to determine any differences between pre- and post-test results in each group. The $95 \%$ confidence interval and percentage of changes were calculated. Statistical significance was accepted at the $p<0.05$ level. The statistical analysis was performed with the SPSS software, V.21.0.

\section{Ethical approval}

The research related to human use has complied with all the relevant national regulations and institutional policies, has followed the tenets of the Declaration of Helsinki, and has been approved by the ethics

Table 2. Effects of foam rolling on lactate concentration, 20-m sprint, and agility $t$-test

\begin{tabular}{|c|c|c|c|c|c|c|c|c|c|c|c|}
\hline \multirow[b]{2}{*}{ Variables } & \multicolumn{4}{|c|}{ FR group } & \multicolumn{4}{|c|}{ SJR group } & \multicolumn{3}{|c|}{$p^{\mathrm{b}}$} \\
\hline & Pre & Post & $\Delta \%$ & $p^{\mathrm{a}}$ & Pre & Post & $\Delta \%$ & $p^{\mathrm{a}}$ & Group & Time & $\begin{array}{l}\text { Group } \\
\times \text { time }\end{array}$ \\
\hline Pre-test lactate $(\mathrm{mmol} / \mathrm{l})$ & $1.79 \pm 0.76$ & $1.99 \pm 0.47$ & 11.20 & 0.135 & $1.92 \pm 0.49$ & $2.45 \pm 0.72$ & 27.80 & $0.016^{*}$ & 0.067 & $0.026^{*}$ & 0.304 \\
\hline 20-m sprint (s) & $4.37 \pm 0.55$ & $4.54 \pm 0.74$ & 4.00 & 0.144 & $4.49 \pm 0.47$ & $4.81 \pm 0.87$ & 7.30 & 0.069 & 0.266 & 0.158 & 0.663 \\
\hline Agility $t$-test (s) & $12.43 \pm 0.75$ & $12.88 \pm 0.72$ & 3.60 & $0.032 *$ & $12.29 \pm 0.60$ & $13.13 \pm 0.88$ & 6.80 & $0.004^{*}$ & 0.769 & $0.001^{*}$ & 0.311 \\
\hline Post-test lactate (mmol/l) & $10.37 \pm 1.67$ & $11.11 \pm 1.42$ & 7.10 & $0.012^{*}$ & $11.03 \pm 1.48$ & $12.01 \pm 0.73$ & 9.00 & $0.024^{*}$ & $0.031 *$ & $0.018 *$ & 0.728 \\
\hline Post-recovery lactate (mmol/l) & $4.57 \pm 1.01$ & $3.96 \pm 0.54$ & -13.28 & $0.033^{*}$ & $5.94 \pm 1.32$ & $6.86 \pm 0.59$ & 15.49 & 0.058 & $0.001^{*}$ & 0.514 & $0.002^{*}$ \\
\hline
\end{tabular}

FR - foam rolling recovery, SJR - slow jogging recovery

${ }^{\mathrm{a}}$ independent $t$-test, ${ }^{\mathrm{b}} 2 \times 2$ repeated measures analysis of variance,

* values significantly different between the groups $(p<0.05)$ 


\section{HUMAN MOVEMENT}

R. Pelana et al., Effects of foam rolling in futsal players

Table 3. Specific changes in lactate concentration, 20-m sprint, and agility $t$-test measures in the FR and SJR groups

\begin{tabular}{|c|c|c|c|c|c|c|c|c|c|c|}
\hline \multirow{2}{*}{ Groups } & \multirow{2}{*}{ Variables } & \multirow{2}{*}{ Time } & \multirow{2}{*}{ Mean } & \multirow{2}{*}{$\begin{array}{l}\text { Standard } \\
\text { deviation }\end{array}$} & \multirow{2}{*}{$\begin{array}{c}\text { Standard } \\
\text { error } \\
\text { mean }\end{array}$} & \multicolumn{2}{|c|}{$\begin{array}{c}\text { 95\% confidence } \\
\text { interval of difference }\end{array}$} & \multirow{2}{*}{$t$} & \multirow{2}{*}{$d f$} & \multirow{2}{*}{$\begin{array}{c}\text { Significance } \\
\text { (2-tailed) }\end{array}$} \\
\hline & & & & & & Lower & Upper & & & \\
\hline FR group & $\begin{array}{l}\text { Pre-test lactate } \\
(\mathrm{mmol} / \mathrm{l})\end{array}$ & Pre-post & -0.20000 & 0.48844 & 0.12611 & -0.47049 & 0.07049 & -1.586 & 14 & 0.135 \\
\hline SJR group & $\begin{array}{l}\text { Pre-test lactate } \\
(\mathrm{mmol} / \mathrm{l})\end{array}$ & Pre-post & -0.53333 & 0.75656 & 0.19534 & -0.95230 & -0.11437 & -2.730 & 14 & $0.016^{*}$ \\
\hline FR group & 20-m sprint (s) & Pre-post & -0.17333 & 0.43337 & 0.11190 & -0.41333 & 0.06666 & -1.549 & 14 & 0.144 \\
\hline SJR group & 20-m sprint (s) & Pre-post & -0.32667 & 0.64195 & 0.16575 & -0.68216 & 0.02883 & -1.971 & 14 & 0.069 \\
\hline FR group & Agility $t$-test (s) & Pre-post & -0.44667 & 0.72493 & 0.18718 & -0.84812 & -0.04521 & -2.386 & 14 & $0.032^{*}$ \\
\hline SJR group & Agility $t$-test (s) & Pre-post & -0.84000 & 0.94627 & 0.24433 & -1.36403 & -0.31597 & -3.438 & 14 & $0.004^{*}$ \\
\hline FR group & $\begin{array}{l}\text { Post-test lactate } \\
(\mathrm{mmol} / \mathrm{l})\end{array}$ & Pre-post & -0.74000 & 0.99197 & 0.25612 & -1.28933 & -0.19067 & -2.889 & 14 & $0.012^{*}$ \\
\hline SJR group & $\begin{array}{l}\text { Post-test lactate } \\
(\mathrm{mmol} / \mathrm{l})\end{array}$ & Pre-post & -0.98800 & 1.51389 & 0.39089 & -1.82637 & -0.14963 & -2.528 & 14 & $0.024 *$ \\
\hline FR group & $\begin{array}{l}\text { Post-recovery } \\
\text { lactate }(\mathrm{mmol} / \mathrm{l})\end{array}$ & Pre-post & 0.60667 & 0.99460 & 0.25681 & 0.05587 & 1.15746 & 2.362 & 14 & $0.033^{*}$ \\
\hline SJR group & $\begin{array}{l}\text { Post-recovery } \\
\text { lactate }(\mathrm{mmol} / \mathrm{l})\end{array}$ & Pre-post & -0.92000 & 1.72262 & 0.44478 & -1.87396 & 0.03396 & -2.068 & 14 & 0.058 \\
\hline
\end{tabular}

FR - foam rolling recovery, SJR - slow jogging recovery

* significant difference between pre- and post-test values $(p<0.05)$

committee of the State University of Jakarta (370/ UN41.14/PR.10/2018).

\section{Informed consent}

Informed consent has been obtained from all individuals included in this study.

\section{Results}

The mean and $S D$ values, as well as percentage of changes for the 20-m sprint, agility $t$-test, and blood lactate concentrations before the test, after the test, and after recovery in the FR and SJR groups are presented in Table 2 .

\section{Blood lactate}

The independent $t$-test revealed no significant differences between the pre- and post-test values in the FR group ( $p=0.135)$ but significant differences were observed between pre- and post-test values in the SJR group ( $p=0.016$ ) for the pre-test lactate (Table 3 ). ANOVA showed a significant main effect for time ( $p=$ 0.026), but no significant main effect was detected for group $(p=0.067)$ or time $\times$ group interactions $(p=$ $0.304)$. For post-test lactate, the independent $t$-test revealed significant changes between pre- and post-test values in the FR and SJR groups $(p=0.012, p=0.024$, respectively). Additionally, ANOVA indicated no significant time $\times$ group interactions $(p=0.728)$. However, the statistical analysis established a significant main effect for group ( $p=0.031)$ and time $(p=0.018)$. Furthermore, for post-recovery lactate, the independent $t$-test revealed a significant decrease between pre- and post-test values in the FR group ( $p=0.033)$. Conversely, an increase between pre- and post-test lactate occurred in the SJR group ( $p=0.058)$. There was no significant main effect for time ( $p=0.514)$ but ANOVA revealed significant time $\times$ group interactions $(p=0.002)$ and a significant main effect for group ( $p=0.001)$.

\section{0-m sprint}

Regarding the 20-m sprint performance, no significant difference was seen for time $(p=0.158)$ or group main effects $(p=0.266)$; no time $\times$ group interaction effects $(p=0.663)$ were present, either. Furthermore, the independent $t$-test revealed no significant changes between pre- and post-test values in the FR and SJR groups ( $p=0.144, p=0.069$, respectively).

\section{Agility $t$-test}

For the agility $t$-test, the independent $t$-test detected a significant increase between pre- and post-test values in the FR and SJR groups ( $p=0.032, p=0.004$, re- 
spectively). Furthermore, ANOVA revealed a significant main effect for time ( $p=0.001)$. Despite, the statistical analysis implied no significant main effect for group $(p=0.769)$ and no significant time $\times$ group interaction $(p=0.311)$.

\section{Discussion}

The aim of the study was to examine whether FR which consisted of 5 exercises, for quadriceps, hamstrings, adductors, gluteal, and gastrocnemius, was an effective tool to aid in the recovery of male professional futsal players. To our knowledge, this is the first study to investigate the influence of FR in elite futsal players. The main findings were the following: (1) FR did not enhance the performance of the agility $t$-test or the 20-m sprint in futsal players; (2) FR can reduce blood lactate concentration better than SJR. The results of this study are important as the selection of recovery methods is crucial in futsal [22].

In the present investigation, the agility $t$-test and the 20-m sprint did not show significant improvements in the FR group, which indicates that FR may not influence performance. The results support two previous studies, which stated that FR did not benefit performance. Healey et al. [17] reported that there was no improvement in athletic performance (vertical jump height and power, isometric force, and pro-agility test) after using the recovery method of FR (comparisons between a bio-foam roller, a uniform polystyrene foam cylinder and multilevel rigid roller, a nonuniform cylinder consisting of a hollow polyvinyl chloride inner core).

In addition, D'Amico and Paolone [23] indicated that FR between two $800-\mathrm{m}$ runs separated by $30 \mathrm{~min}$, which consisted of 5 exercises (for gluteal muscles, hip flexors, quadriceps, iliotibial bands, adductors, and calves), did not alter performance in trained male runners. For these phenomena, we may speculate that FR did not bring physiological effects on the musculature. In contrast, Phillips et al. [24] reported that pro-agility performance improved slightly after $1 \mathrm{~min}$ of continuous self-myofascial release with a foam roller in recreationally active males and females. These studies may have differed from our research because the optimal protocols to assist FR for exercise performance remain unalike. Various types of roller massagers, timing, history of activity in the sample, foam roll intervention, and activity level can all vary, causing differences in results.

An important finding of this study is the effect of FR on blood lactate concentration. Specifically, the study showed that FR was more effective in reducing blood lactate concentration in male futsal athletes compared with SJR after muscle fatigue due to exercise. Our findings coincided with those reported by Kalén et al. [25], who indicated that surf lifesavers cleared out blood lactate more efficiently when performing an FR protocol than with passive recovery. Although the underlying mechanisms supporting the decrease in blood lactate concentration as a consequence of FR remain unclear, a few previous studies suggested that FR exercises could induce structural, metabolic, or neural alterations that led to changes in delayed-onset muscle soreness, decreased oedema, enhanced blood lactate removal, and improved tissue healing, mainly owing to an increase in muscular blood flow [20, 26].

Additionally, previous literature explained the physiological mechanism of FR; namely, during FR exercise, a high mechanical load to the entire underlying tissue is induced, potentially leading to increased blood flow [27]. In turn, increased blood flow can hinder the margination of neutrophils and reduce prostaglandin production, subsequently decreasing inflammation [28]. Furthermore, a systematic review implied that the methods of FR myofascial release induced muscular blood flow and resulted in increased oxygen delivery, which encouraged mitochondrial resynthesis of adenosine triphosphate and the active transport of calcium back into the sarcoplasmic reticulum [29]. Because of this physiological mechanism, we may speculate that the action of FR can reduce blood lactate concentration in futsal athletes.

On the other hand, previous literature also described negative effects of FR. Freiwald et al. [30] stated that FR exercises could potentially lead to harmful effects on connective tissue, nerves, vessels, and bones, caused by a high mechanical load in the entire underlying tissue. Although we are limited to commenting on the negative effect of FR as a tool to assist recovery in elite futsal athletes, we suggest that coaches, athletes, and sports scientists should examine the FR frequency, intensity (amount of pressure placed on the foam roller), time (immediately after exercise vs. at other time points), and type (sweeping pressure vs. undulations) to avoid harmful factors caused by the application of FR.

Briefly, our results have indicated that post-training FR exercises may aid to reducing blood lactate concentration but did not enhance the performance of the agility $t$-test or the 20 -m sprint in futsal players. The results of this study can support previous scientific literature which states that FR can provide benefits in the post-exercise recovery process and suggests 
that FR consisting of 5 exercises (for quadriceps, hamstrings, adductors, gluteal, and gastrocnemius) can assist recovery after exercise-induced muscle fatigue. However, future studies are still needed to explore a number of issues that could not be addressed in this study.

\section{Limitations}

The authors realize that there are some limitations in this study that must be considered in future research. Firstly, we did not evaluate the impact of the participants' lifestyle or emotional functioning, which might have influenced the results. Secondly, the testing period may have been unbalanced between the FR and SJR groups, with the former group being faster than the latter, but this study has succeeded in showing differences in the two recovery modalities. Thirdly, we recognize the need to apply other measurement tools in future studies, such as the total quality recovery scale or visual analogue scale, in order to answer questions that have not been resolved in this study.

\section{Conclusions}

The results of this study indicate that FR after exercise-induced muscle fatigue, which consisted of 5 exercises (for quadriceps, hamstrings, adductors, gluteal, and gastrocnemius), had an effect on the recovery of elite futsal players by increasing the rate of blood lactate clearance, but no increase was observed in the performance of the 20-m sprint or the agility $t$-test. Thus, we suggest that coaches or sports scientists should recommend FR rather than SJR to their athletes for reducing blood lactate concentration after futsal training sessions.

\section{Acknowledgments}

The authors would like to thank the Bandung Institute of Technology and State University of Jakarta for providing the means for data retrieval. The study was not supported financially by any agency.

\section{Disclosure statement}

No author has any financial interest or received any financial benefit from this research.

\section{Conflict of interest}

The authors state no conflict of interest.

\section{References}

1. De Moura NR, Borges LS, Santos VC, Joel GB, Bortolon JR, Hirabara SM, et al. Muscle lesions and inflammation in futsal players according to their tactical positions. J Strength Cond Res. 2013;27(9):26122618; doi: 10.1519/JSC.0b013e31827fd835.

2. Doğramacı NS, Watsford LM. A comparison of two different methods for time-motion analysis in team sports. Int J Perform Anal Sport. 2006;6(1):73-83; doi: 10.1080/24748668.2006.11868356.

3. Castagna C, D’Ottavio S, Vera JG, Álvarez JCB. Match demands of professional futsal: a case study. J Sci Med Sport. 2009;12(4):490-494; doi: 10.1016/j.jsams.2008. 02.001 .

4. Barbieri RA, Zagatto AM, Milioni F, Barbieri FA. Specific futsal training program can improve the physical performance of futsal players. Sport Sci Health. 2016; 12(2):247-253; doi: 10.1007/s11332-016-0283-z.

5. Weerapong P, Hume PA, Kolt GS. The mechanisms of massage and effects on performance, muscle recovery and injury prevention. Sports Med. 2005;35(3):235256; doi: 10.2165/00007256-200535030-00004.

6. Ream E, Richardson A. Fatigue: a concept analysis. Int J Nurs Stud. 1996;33(5):519-529; doi: 10.1016/ 0020-7489(96)00004-1.

7. Finsterer J. Biomarkers of peripheral muscle fatigue during exercise. BMC Musculoskelet Disord. 2012; 13(1):218; doi: 10.1186/1471-2474-13-218.

8. Van Hall G. Lactate kinetics in human tissues at rest and during exercise. Acta Physiol. 2010;199(4):499508; doi: 10.1111/j.1748-1716.2010.02122.x.

9. Barnett A. Using recovery modalities between training sessions in elite athletes: does it help? Sports Med. 2006;36(9):781-796; doi: 10.2165/00007256-200636 090-00005.

10. Kellmann M, Bertollo M, Bosquet L, Brink M, Coutts AJ, Duffield R, et al. Recovery and performance in sport: consensus statement. Int J Sports Physiol Perform. 2018;13(2):240-245; doi: 10.1123/ijspp.2017-0759.

11. Rey E, Padrón-Cabo A, Barcala-Furelos R, Casamichana D, Romo-Pérez V. Practical active and passive recovery strategies for soccer players. Strength Cond J. 2018; 40(3):45-57; doi: 10.1519/SSC.0000000000000247.

12. Hemmings TJ, Kendall KL, Dobson JL. Identifying dosage effect of light-emitting diode therapy on muscular fatigue in quadriceps. J Strength Cond Res. 2017; 31(2):395-402; doi: 10.1519/JSC.0000000000001523.

13. Argus CK, Broatch JR, Petersen AC, Polman R, Bishop DJ, Halson S. Cold-water immersion and contrast water therapy: no improvement of short-term recovery after resistance training. Int J Sports Physiol Perform. 2017;12(7):886-892; doi: 10.1123/ijspp.2016-0127.

14. Rey E, Lago-Peñas C, Lago-Ballesteros J, Casáis L. The effect of recovery strategies on contractile properties using tensiomyography and perceived muscle soreness in professional soccer players. J Strength Cond Res. 2011;26(11):3081-3088; doi: 10.1519/JSC.0b013e318 $2470 \mathrm{~d} 33$. 
15. Schroeder AN, Best TM. Is self myofascial release an effective preexercise and recovery strategy? A literature review. Curr Sports Med Rep. 2015;14(3):200-208; doi: 10.1249/JSR.0000000000000148.

16. Barnes MF. The basic science of myofascial release: morphologic change in connective tissue. J Bodyw Mov Ther. 1997;1(4):231-238; doi: 10.1016/S1360-8592 (97)80051-4.

17. Healey KC, Hatfield DL, Blanpied P, Dorfman LR, Riebe D. The effects of myofascial release with foam rolling on performance. J Strength Cond Res. 2014; 28(1):61-68; doi: 10.1519/JSC.0b013e3182956569.

18. Rey E, Padrón-Cabo A, Costa PB, Barcala-Furelos R. Effects of foam rolling as a recovery tool in professional soccer players. J Strength Cond Res. 2019;33(8):21942201; doi: 10.1519/JSC.0000000000002277.

19. Madoni SN, Costa PB, Coburn JW, Galpin AJ. Effects of foam rolling on range of motion, peak torque, muscle activation, and the hamstrings-to-quadriceps strength ratios. J Strength Cond Res. 2018;32(7):1821-1830; doi: 10.1519/JSC.0000000000002468.

20. Pearcey GEP, Bradbury-Squires DJ, Kawamoto J-E, Drinkwater EJ, Behm DG, Button DC. Foam rolling for delayed-onset muscle soreness and recovery of dynamic performance measures. J Athl Train. 2015; 50(1):5-13; doi: 10.4085/1062-6050-50.1.01.

21. Macdonald GZ, Button DC, Drinkwater EJ, Behm DG. Foam rolling as a recovery tool after an intense bout of physical activity. Med Sci Sports Exerc. 2014;46(1): 131-142; doi: 10.1249/MSS.0b013e3182a123db.

22. Nakamura FY, Antunes P, Nunes C, Costa JA, Esco MR, Travassos B. Heart rate variability changes from traditional vs. ultra-short-term recordings in relation to preseason training load and performance in futsal players. J Strength Cond Res. 2018; doi: 10.1519/JSC.00000 00000002910.

23. D’Amico A, Paolone V. The effect of foam rolling on recovery between two eight hundred metre runs. J Hum Kinet. 2017;57(1):97-105; doi: 10.1515/hukin-2017-0051.

24. Phillips J, Diggin D, King DL, Sforzo GA. Effect of varying self-myofascial release duration on subsequent athletic performance. J Strength Cond Res. 2018; doi: 10.1519/JSC.0000000000002751.

25. Kalén A, Pérez-Ferreirós A, Barcala-Furelos R, Fernández-Méndez M, Padrón-Cabo A, Prieto JA, et al. How can lifeguards recover better? A cross-over study comparing resting, running, and foam rolling. Am J Emerg Med. 2017;35(12):1887-1891; doi: 10.1016/j.ajem.2017.06.028.

26. Casanova N, Reis JF, Vaz JR, Machado R, Mendes B, Button DC, et al. Effects of roller massager on muscle recovery after exercise-induced muscle damage. J Sports Sci. 2018;36(1):56-63; doi: 10.1080/02640414.2017. 1280609 .

27. MacDonald GZ, Penney MDH, Mullaley ME, Cuconato AL, Drake CDJ, Behm DG, et al. An acute bout of self-myofascial release increases range of motion without a subsequent decrease in muscle activation or force.
J Strength Cond Res. 2013;27(3):812-821; doi: 10.1519/ JSC.0b013e31825c2bc1.

28. Smith LL, Keating MN, Holbert D, Spratt DJ, McCammon MR, Smith SS, et al. The effects of athletic massage on delayed onset muscle soreness, creatine kinase, and neutrophil count: a preliminary report. J Orthop Sports Phys Ther. 1994;19(2):93-99; doi: 10.2519/jospt.1994.19.2.93.

29. Beardsley C, Škarabot J. Effects of self-myofascial release: a systematic review. J Bodyw Mov Ther. 2015; 19(4):747-758; doi: 10.1016/j.jbmt.2015.08.007.

30. Freiwald J, Baumgart C, Kühnemann M, Hoppe MW. Foam-rolling in sport and therapy - potential benefits and risks: Part 2 - Positive and adverse effects on athletic performance. Sports Orthop Traumatol. 2016;32(3): 267-275; doi: 10.1016/j.orthtr.2016.07.002. 